\title{
CHOICE OF TREATMENT OPTIONS FOR CYSTIC LYMPHSTIC MALFORMATIONS IN THE HEAD AND NECK REGION: TREATMENT EXPERIENCE OF 81 CHILDREN
}

Background. Surgery has previously been the only treatment for lymphatic malformations (LMs), but in the head and neck region is challenging due to the risk of scarring, nerve damage, recurrence. Sclerotherapy may be a perfect alternative.

Objective. The aim of the study is to determine the efficacy and safety of the OK-432 sclerotherapy in the children with craniofacial LMs.

Methods. 81 children with head and neck LM between December 2010 and March 2017 were involved into the study. The follow-up period was from 6 to 79 months. According to the size of cysts, LMs were classified into macrocystic, microcystic, and mixed. The result of the treatment of LMs was determined by the percentage of reduction in size as excellent (decrease by more than 90\%), good (by 50\%-89\%), satisfactory (by 20\%-49\%) and none (by less than 20\%).

Results. The macrocystic LMs diagnosed in $41.97 \%$ of patients, microcystic - in $12.35 \%$, and mixed - in $45.68 \%$ of children. OK-432 sclerotherapy only was performed for $83.9 \%$ of patients and in $12.3 \%$ in combination with surgery. The range of sclerotherapy sessions was from 1 to 11. An excellent result in $96.97 \%$ of cases was evidenced in the patients with macrocystic LM. Poor result was proved in the patients with microcystic LMs; the most of them (55.56\%) had satisfactory result. In the patients with mixed $L M$, an excellent and good result was evidenced in $83.33 \%$. After 198 sessions of OK-432 sclerotherapy, complications associated with the treatment occurred in 5 (2.52\%) cases.

Conclusions. OK-432 sclerotherapy is a safe and effective treatment of head and neck LMs in children. Macrocystic LMs proved the best response to OK-432 treatment.

KEY WORDS: lymphatic malformations; sclerotherapy; OK-432; children.

\section{Introduction}

Cystic lymphatic malformations are benign vascular lesions, which develop as the result of embryological disturbances of lymphatic system. Unfortunately, the terms often used to describe LMs are imprecise. 'Cystic hygroma' and 'lymphangioma' are often used incorrectly to describe malformations. Both of these terms should be abandoned, as the suffix -oma connotes a neoplasm [1]. In 1982, a landmark article by Mulliken and Glowacki suggested $a$ classification system designating vascular anomalies as either tumours or malformations according to their biologic and pathologic features [2]. This system was adopted by the international society for the study of vascular anomalies (ISSVA) and was last updated in 2014

Corresponding author: Iryna Benzar, Department of Pediatric Surgery, Bogomolets National Medical University, 28/1 V. Chornovola, Kyiv, Ukraine, 01135

Phone number: +380951295882

E-mail:iryna.benzar@nmu.ua,ira_benzar@yahoo.com
[3]. Vascular malformations are classified as capillary, venous, lymphatic, arterial, and mixed lesions, depending on their vascular tissue of origin. These lesions are present at birth and have a progressive clinical course. The incidence of LMs is evidenced in about 1 to 6000 to 1 to 16,000 live births, with a frequency of hospitalization - 3 cases per 100,000 [4, 5]. LMs near the principal lymphatic chains in the neck region are formed when a primordial lymph sac loses or fails to re-establish communication with the central veins (jugular and subclavian) from which it arises [1]. For many years, surgery has been the only treatment for LMs of any anatomical localization. However, the results of the operation were often disappointing, especially in cases of LM of cheek, tongue, oral cavity, neck lateral triangle. The surgical treatment of LM in the region of head and neck is accompanied by complications in $12-33 \%$ and recurrences in $15-53 \%$ of cases. In addition, 
resection of LM does not inhibit bones overgrowth in the region of LMs $[6,7,8]$. Recurrence and complications are usually the result of incomplete preoperative examination and incorrectly planned treatment. Incomplete resection provokes rapid growth of malformation, causes postoperative lymphorrhea, and recurrent infections [9]. The role of surgical treatment in order to achieve an excellent cosmetic result in patients with LMs is questionable. When planning an intervention, it is necessary to clearly understand that LM can sprout fascia, infiltrate different tissues and change normal anatomy. The damage of important nervous and vascular structures is unacceptable in the treatment of benign pathology [10]. LMs usually infiltrate skin and subcutaneous tissue [1], therefore after the operation the asymmetry progresses, and later lymph nodes can sprout post-operative scars.

Currently, there is no sufficient evidence in the literature to create treatment algorithms and help determine the best choice of initial therapy, and in recent decades sclerotherapy has become the most common treatment option $[5,11,12]$.

According to the results of the literature analysis in the PubMed, Medline, Cochrane databases, three therapeutic agents are widely used for cystic LM local treatment: bleomycin, doxycycline and OK-432 (Picibanil) [13]. The features of endothelial cells lining the LMs cysts determine the effectiveness of the OK-432 for the treatment of LM. The mechanism of action of the drug is based on the induction of inflammation with subsequent activation of cytokines and apoptosis of cells that form the inner layer of the cysts [14]. In the cyst fluid the level of inflammatory mediators increases that confirms the inflammation in response to the injection of the drug [15]. The effectiveness of the OK-432 sclerotherapy in the treatment of cystic LMs has been described in small series of some medical centres $[16,17]$. Since 2011 OK-432 is successfully used in Ukraine [18].

Objective of the study is to analyse the treatment experience of 81 child patients with lymphatic malformations (LM) in the head and neck region, and to determine the efficacy and safety of the OK-432 sclerotherapy in this cohort of patients.

\section{Methods}

During the period from December 2010 to March 2017, 126 patients with isolated LMs or LMs in combination with other congenital vascular malformations were treated in the OKHMATDYT National Specialized Children Hospital. The study includes 81 clinical cases of LMs that are localized in the head and neck region, representing $64.29 \%$ of all patients with LMs during the established period of time. The follow up period was from 6 to 79 months. There were $44(53.7 \%)$ male and 37 (45.1\%) female patients.

The following visualization methods were used to confirm the diagnosis of cystic LMs: ultrasound scan in grey-scale and colour Doppler was performed at the primary patient's examination and in dynamics, MRI was performed before treatment and at the stages of treatment, CT in urgent situation. According to the results of MRI, the size and structure of LMs and also their topographical relationship with neighbouring organs and tissues were determined as particularly mediastinum with retropharyngeal space involvement associated with LMs to the level of the hyoid bone. LMs were classified as macrocystic, microcystic, or mixed (macrocysticmicrocystic), which has been proven useful in other sclerotherapystudies [19]. The classification was determined radiographically by MRI and ultrasound examination according to the cysts size: macrocystic (formed by cysts with a volume of more than 2 cubic centimetres (cc)), microcystic (cysts volume - less than $2 \mathrm{cc}$ ), and mixed, in which the microcystic component exceeds $50 \%$. The size of LMs was determined using the ellipse square formula. The size of LMs up to $100 \mathrm{~cm}^{2}$ was evaluated as small, 100-199 $\mathrm{cm}^{2}$ - middle, 200-299 $\mathrm{cm}^{2}$ - large and more than $300 \mathrm{~cm}^{2}-$ gigantic. The result of the treatment of LM was determined by the percentage of reduction in the lesion size $[3,10]$ as excellent (decrease by more than $90 \%)$, good (50-89\%), satisfactory $(20-49 \%)$ and unsatisfactory (less than $20 \%$ reduction in size).

The statistical analysis was performed using IBM SPSS Statistics, version 23. The data is presented as an average of 95 percent confidence interval or standard deviation. The qualitative data (age, sex, localization, size, etc.) were analysed in a univariate analysis with the Pearson's correlation coefficient $\chi^{2}$ test. The logistic regression model was used for variants that significantly predict the outcome of the treatment and/or the course of the disease. Statistical significance was defined as $p<0.05$.

\section{Results}

Diagnosis of LMs were established prenatally in $12(14.81 \%)$ patients, at birth in $43(53.08 \%)$, 
during the first year of life in 12 children $(14.81 \%)$, at the age of one to three years in 4 $(4,94 \%)$ and in $10(12.35 \%)$ children, the clinical signs of the disease were manifested at the age above three years. Clinical signs of cystic LM in the head and neck region were: the asymmetry of face and neck due to mass in all children, compression of upper airway requiring tracheostomy before sclerotherapy $(n=4,3.6 \%)$, transient stridor during the first year of life $(n=8$, $7,1 \%)$, macroglossia, disarticulation $(n=5,4,5 \%)$, disturbance of occlusion $(n=6,5,4 \%)$, recurrence lymphorrhoea in the children with mucosal and skin lesions $(n=4,3.6 \%)$, excessive salivation $(n=3,2.7 \%)$, visual impairment $(n=1,0.9 \%)$, lymphedema of upper $\operatorname{limb}(n=1,0.9 \%) .68$ patients were treated primary, 13 children underwent surgical interventions previously. There patients were treated conservatively with propranolol within 6-12 months without any clinical result. According to the visualization of LMs using ultrasound and MRI, the topographic features of LM and relationship to adjacent organs were established. The lesions were localized in suprahyoid region in 37 (45.68\%) children, and in infrahyoid region - in 44 $(54.32 \%)$ cases. Bilateral mass were observed in $23(28.39 \%)$ children, in other patients LMs were localized on the one side of middle line. $34(41.97 \%)$ patients had macro cystic LMs, in 10 (12.35\%) children microcytic LMs were diagnosed, and in 37 (45.68\%) children LMs were mixed. The size of LMs ranged from 23 to $517 \mathrm{~cm}^{2}$, in average $135.87 \mathrm{~cm}^{2}$. In $37(45.68 \%)$ patients LMs were small; in 29 (35.80\%) they were middle, in 7 (8.64\%) LMs were large and in $8(9.88 \%)$ LMs were gigantic. In $22(2716 \%)$ children mediastinal involvement was diagnosed. In children with head and neck LMs sclerotherapy, surgical and combined treatments were performed.

Three patients (3.7\%) underwent surgical resection of LMs for the following reasons: recurrent inflammation requiring re-hospitalization and antibiotic therapy $(n=2)$, difficulty in diagnostic due to sudden onset and atypical course of the disease $(n=1)$. Recurrence after surgery happened in one case. One patient had postoperative complication: transient paresis of facial nerve branch.

Giant LMs, which occupy several anatomical sites and infiltrate muscles, bones, cellular spaces deserved a particular attention. Bilateral LMs narrowed airway and tracheostomy was evidenced in 5 patients. In this series, decannulation was eventually possible in 4 patients after multiple sessions of OK-432 sclerotherapy. One patient with bilateral LMs, mediastinum, retropharyngeal space and lung involvement died due to septic complications. Combined treatment was performed for $10(12.3 \%)$ children, which consisted of the following procedures: resection of LM, tracheostomy prior to treatment, injection of OK-432 into residual cyst cavity during and/or after surgery $(n=5,6.17 \%)$, thoracocentesis, pleural and mediastinal drainage in the patient with purulent mediastinitis with subsequent sclerotherapy after the elimination of complications $(n=1)$, sclerotherapy and correction of scarring and tissue deformities $(n=3)$, resection of tongue in the patient with pronounced macroglossia, disturbance of occlusion $(n=1)$, resection of orbital part of LMs due to visual impairment and subsequent sclerotherapy $(n=1)$. The most patients $(n=68,83.90 \%)$ underwent the OK-432 sclerotherapy as a single method of head and neck LMs. Patients received from 1 to 11 sessions of sclerotherapy, in average $2.44 \pm 2.21$ sessions per patient. After 1 session of the OK-432 sclerotherapy, an excellent result was achieved in $24(29.63 \%)$ patients, according to localization and structure, these were unilateral macro cystic LMs of neck. Involvement of the mediastinum and retropharyngeal space was not a contraindication to the sclerotherapy of LMs. In 18 children with mediastinal and retropharyngeal part of LMs an excellent result was evidenced in $5(27.78 \%)$, good in $9(50.0 \%)$, and satisfactory in $2(11.11 \%)$ cases. However, in 1 case, the result was poor and in 1 case the result of the treatment was unsatisfactory.

After 198 sessions of the OK-432 sclerotherapy, complications associated with the treatment occurred in 5 (2.52\%) cases: significant oedema with the need for hospitalization, puncture, and decompression of the cyst $(n=4$; $2.02 \%)$, and skin allergic reaction $(n=1 ; 0.51 \%)$. Response data for macro cystic LMs were higher than in other types of LMs with an excellent result in $96.97 \%$ and good result in 3.03\% of patients. In the patients with microcystic LMs, no positive result was evidenced, $33.33 \%$ of cases had a good treatment result, $55.56 \%$ were satisfactory and $11.11 \%$ had no results. In the patients with mixed LM, an excellent result was evidenced in $33.33 \%$ of cases, $50.0 \%$ of patients had good response to treatment, $11.11 \%-\mathrm{a}$ satisfactory one, and $2.77 \%$ of patients had no result.

Primary efficacy endpoints were evaluated using Fisher's exact test. Covariates used in a 
univariate analysis included: LM type (macrocystic, microcystic, mixed), laterality (unilateral versus bilateral), mediastinal and retropharyngeal involvement, prior treatment, age during the first treatment, gender. It was established, that the result of treatment was determined primarily by the type of LM and previous intervention: the best results were evidenced in the patients with macrocystic LMs $(p<0.005)$, previous intervention reduced the treatment efficacy $(p<0.005)$. Covariates that proved a significant association with the treatment result were used for logistic regression analysis, the probability of an excellent and good outcome for the patients with macrocystic LM in cases of no prior treatment was $86.6 \%$.

\section{Discussion}

This article presents a relatively large experience of the treatment of cystic LMs in the head and neck region for a relatively short period of time (5.5 years). A peculiarity of this study is that for the first time in Ukraine a new approach to the treatment of LMs of challenging localization in children is presented. Treatment of head and neck LMs is accompanied by significant risks, therefore for children it is necessary to choose the most effective and safe way, until stronger evidence is present, the difference in complication rates is potentially the deciding factor in the choice the treatment options [13].

For decades, resection has been the mainstay for management of LMs. More recently, sclerotherapy has become the first-line treatment for head and neck lesions. Experienced surgeons welcome the ascendancy of sclerosant management. Although some LMs can be successfully resected, complications are expected and results are often disappointing.

One of the most safe and effective ways of cystic LMs treatment in children is the OK-432 sclerotherapy $[5,16]$. The OK-432 (Chugai Pharmaceuticals, Tokyo, Japan) is a lyophilized powder of Streptococcus pyogenes (group A, type 3, Su strain) incubated with benzylpenicillin. The drug was developed in Japan in the late 1960 s as an antitumor agent. Although therapy of OK-432 did not increase the survival of the patients with cancer, its efficacy was proved in pleurodesis in cases of malignant pleural involvement. According to these studies, in the late 1980s, Japanese authors [17] published the first results of using OK-432 as a safe and effective treatment for cystic LMs; in 1994, the authors published the results of treatment of 94 patients with LMs [19]. Since then, the method has become widely used far beyond the borders of the country $[20,21]$. The OK-432 is not exactly a sclerosing agent, because it doesn't destroy vascular endothelium. The OK432 induces apoptosis of lymphatic endothelium and local cellular inflammatory reaction $[7,16]$. Recent studies have proved that the pathway of the OK-432 action within lymphangiomas is probably cellular and cytokine-mediated $[7,16]$.

Complications due to OK-432 management are temporary and predictable [22]. According to the results of the literature analysis, no systemic hematological, renal, hepatic or cardiac side effects were detected [23], which was confirmed in our study. The typical side effect of OK-432 sclerotherapy is an inflammatory response that is accompanied by local oedema and may be potentially dangerous in cases of an airway compromised by cervical LMs. In our series, in the patients with impaired mechanics of breathing, tracheostomy was performed prior to treatment. All of those children had signs of respiratory failure, tracheostomy as a preventive procedure was not performed.

Unfortunately, none of the suggested methods for cystic LM treatment in children guarantees the complete recovery for all patients [24]; however, the introduction of a minimally invasive treatment of LM significantly reduced the percentage of open surgical interventions and made it possible to differentiate treatment in each individual clinical case.

\section{Conclusions}

The OK-432 sclerotherapy is safe and effective treatment option of head and neck LMs in children. Macrocystic LMs prove the best response to the OK-432 treatment, previous intervention and increasing the part of microcystic component reduces the efficacy of treatment. Complications associated with the OK432 treatment occurred in $2.52 \%$ cases.

\section{References}

1. Elluru R, Balakrishnan K, Padua H. Lymphatic malformations: Diagnosis and management. Semin
Pediatr Surg. 2014;23(4):178-85. doi: 10.1053/j. sempedsurg.2014.07.002. 
2. Mulliken JB, Glowacki J. Hemangiomasand vascular malformations in infants and children: a classification based on endothelial characteristics. Plast Reconstr Surg. 1982;69(3):412-22.

3. Wassef M, Blei F, Adams D, Alomari A, Baselga $E$, Berenstein $A$, et al. Vascular Anomalies Classification: Recommendations From the International Society for the Study of Vascular Anomalies. Pediatrics. 2015 Jul;136(1):e203-14. doi: 10.1542/ peds.2014-3673.

4. Perkins J, Manning S, Tempero R, Cunningham M, Edmonds J, Hoffer F, et al. Lymphatic malformations: Current cellular and clinical investigations. Otolaryngol Head Neck Surg. 2010;142(6):78994. doi: 10.1016/j.otohns.2010.02.025.

5. Churchill P, Otal D, Pemberton J, Ali A, Flageole $\mathrm{H}$, Walton JM. Sclerotherapy for lymphatic malformations in children: a scoping review. J Pediatr Surg. 2011;46(5):912-22. doi: 10.1016/j.jpedsurg.2011.02.027.

6. Chen E, Hostikka S, Oliaei S, Duke W, Schwartz S. Perkins J. Similar Histologic Features and Immunohistochemical Staining in Microcystic and Macrocystic Lymphatic Malformations. Lymphat Res Biol. 2009; 7(2):75-80. doi: 10.1089/Irb.2009.0003.

7. Ardıclı B, Karnak I, Ciftci AO, Tanyel FC, Senocak ME. Sclerotherapy with bleomycin versus surgical excision for extracervical cystic lymphatic malformations in children. Surg Today. 2016;46(1):97-101. 10.1007/s00595-015-1128-0.

8. Weitz-Tuoretmaa A, Rautio R, Valkila J, KeskiSäntti H, Keski-Nisula L, Laranne J. Efficacy of OK-432 sclerotherapy in treatment of lymphatic malformations: long-term follow-up results. Eur Arch Otorhinolaryngol. 2014;271(2):385-90. doi: 10.1007/ s00405-013-2542-9.

9. Adams MT, Saltzman B, Perkins JA. Head and Neck Lymphatic Malformation Treatment. Otolaryngol Head Neck Surg. 2012;147(4):627-39.

10. Love Z. Hsu D. Low-flow vascular malformations of the head and neck: clinicopathology and image guided therapy. J Neurointerv Surg. 2012; 4(6):414-25. doi: 10.1136/neurintsurg-2011-010126.

11. Malic CC, Guilfoyle R, Courtemanche RJM, Arneja JS, Heran MKS, Courtemanche DJ. Lymphatic Malformation Architecture. J Craniofac Surg. 2017; 28(7):1721-1724. doi: 10.1097/SCS.0000000000003789.

12. Acevedo JL, Shah RK, Brietzke SE. Nonsurgical therapies for lymphangiomas: A systematic review. Otolaryngol Head Neck Surg. 2008;138(4):418-24. doi: 10.1016/j.otohns.2007.11.018.
13. Horbach SE, Lokhorst MM, Saeed P, de Goüyon Matignon de Pontouraude CM, Rothová A, van der Horst CM. A. Sclerotherapy for low-flow vascular malformations of the head and neck: A systematic review of sclerosing agents. J Plast Reconstr Aesthet Surg. 2016;69(3):295-304. doi: 10.1016/j.bjps.2015.10.045.

14. Wiegand S, Eivazi B, Sel S, Renz H, Werner JA, Folz BJ. Analysis of Cytokine Levels in Human Lymphangiomas. In Vivo. 2008;22(2):253-6.

15. Ogita S, Tsuto T, Deguchi E, Tokiwa K, Nagashima M, Iwai N. OK 432 therapy for unresectable lymphangiomas in children. J Pediatr Surg. 1991; 26(3):263-8.

16. Ghaffarpour N, Petrini B, Svensson LA, Boman K, Wester T, Claesson G. Patients with lymphatic malformations who receive the immunostimulant OK-432 experience excellent long-term outcomes. Acta Paediatr. 2015;104(11):1169-73. doi: 10.1111/ apa.13086.

17. Ogita S, Tsuto T, Tokiwa K, Takahashi T. Intracystic injection of OK-432: a new sclerosing therapy for cystic hygroma in children. Br J Surg. 1987; 74(8):690-1.

18. Benzar I. Treatment of Lymphatic malformations with OK-432: the First Experience of a Single Hospital. Internat J of Biomed. 2014;4(4):237-41.

19. Ogita S, Tsuto T, Nakamura K, Deguchi E, Iwai N. OK-432 therapy in 64 patients with lymphangioma. J Pediatr Surg. 1994;29(6):784-5.

20. Poldervaart MT, Breugem CC, Speleman L, Pasmans S. Treatment of Lymphatic Malformations With OK-432 (Picibanil). J Craniofac Surg. 2009; 20(4):1159-62. doi: 10.1097/SCS.0b013e3181abb249.

21. Tu JH, Do HM, Patel V, Yeom KW, Teng JMC. Sclerotherapy for lymphatic malformations of the head and neck in the pediatric population. J Neurointerv Surg. 2017;9(10):1023-1026. doi: 10.1136/ neurintsurg-2016-012660.

22. Smith MC, Zimmerman MB, Burke DK, Bauman NM, Sato Y, Smith RJ. Efficacy and safety of OK-432 immunotherapy of lymphatic malformations. Laryngoscope. 2009;119(1):107-15. doi: 10.1002/ lary.20041.

23. Kim DW. OK-432 sclerotherapy of lymphatic malformation in the head and neck: factors related to outcome. Pediatr Radiol. 2014;44(7):857-62. doi: 10.1007/s00247-014-2889-0.

24. Trenor CC 3rd, Chaudry G. Complex lymphatic anomalies. Semin Pediatr Surg. 2014;23(4):186-90. doi: 10.1053/j.sempedsurg.2014.07.006. 